\title{
AVALIAÇÃO DO RISCO PARA A SAÚDE DEVIDO À PRESENÇA DE TRIHALOMETANOS NA ÁGUA DE ABASTECIMENTO
}

\author{
HEALTH RISK ASSESSMENT FROM EXPOSURE TO TRIHALOMETHANES IN \\ DRINKING WATER
}

\author{
António Sampaio Duarte ${ }^{a}$, Filipe Miguel Rodrigues ${ }^{b}$ \\ aUniversidade do Minho, Portugal; 'banco KfW. \\ e-mail: aduarte@civil.uminho.pt, fmiguelrod@gmail.com
}

Submissão: 30 de janeiro de 2020 Aceitação: 22 de junho de 2020

\section{Resumo}

A aplicação de compostos clorados na desinfeção da água pode induzir à formação de subprodutos da desinfeção (SPD) com efeitos carcinogénicos e mutagénicos comprovados. Neste trabalho, apresenta-se uma síntese de um estudo sobre a ocorrência de trihalometanos (THM) na água de abastecimento, realizado nos catorze municípios do distrito de Braga (Portugal), visando a avaliação do risco de cancro $(\mathrm{RC})$ e de efeitos não carcinogénicos (HI) com base na metodologia desenvolvida pela USEPA e IRIS. Num cenário gravoso, correspondente à prevalência da maior concentração de THM detetada no período monitorizado, o indicador RC daria valores superiores ao limite máximo definido como negligenciável, verificando-se ser a ingestão a via de exposição mais relevante. Os resultados obtidos para o indicador $\mathrm{HI}$ foram quase sempre inferiores à unidade, indicando um nível de risco aceitável, de acordo com os pressupostos do método utilizado. O uso de indicadores do risco para a saúde constitui uma ferramenta útil quer no apoio à decisão das entidades gestoras de sistemas de abastecimento de água, quer na definição de estratégias e políticas de saúde pública que visem a mitigação deste risco.

Palavras-chave: Tratamento de água; trihalometanos; avaliação do risco de cancro.

\section{Abstract}

Chlorination have brought enormous benefits to the preservation of public health as a low-cost disinfection method, but it can generate several halogenated organic compounds, as disinfection by-products (DBP), with potential and proven carcinogenic and mutagenic effects. This work presents a synthesis of the study performed in water supply systems of the fourteen municipalities of Braga district (Portugal), aiming at the evaluation of life time cancer risk (CR index), and the risk of non-carcinogenic effects (HI-Hazard Index) from exposure to THMs, using a method proposed by USEPA and IRIS. In a severe scenario, corresponding to the prevalence of maximum THM concentration occurred during the monitored period, the CR index would achieve values higher than the maximum limit defined as negligible for all these municipalities. The $\mathrm{HI}$ index results were often lower than one, which is considered as an acceptable risk level. The use of health risk indicators can be a useful tool both in supporting the decision-making of water supply utilities and in the definition of public health strategies/policies for risk mitigation in order to increase the life quality and the wellness of populations.

Keywords: Drinking water treatment; trihalomethanes; cancer risk assessment.

\section{INTRODUÇÃO}

A concentração das populações em centros urbanos, resultante de fenómenos migratórios e do êxodo das áreas rurais mais subdesenvolvidas, tem vindo a aumentar de forma exponencial (FU et al., 2013), sendo as atuais e futuras megacidades um fator de 
preocupação e um grande desafio em termos de saúde pública. Esse crescente afluxo de populações às cidades, aumentando as densidades demográficas, tem conduzido ao incremento da poluição e contaminação das origens de água, dificultando ainda mais, num contexto de alterações climáticas, a missão de se garantir o acesso universal a uma água segura (DUARTE, 2007; FUNARI et al., 2012), por meio de estratégias de controlo do risco assente numa abordagem de barreiras múltiplas.

A desinfeção aplicada no tratamento de água para consumo humano constituiu um dos maiores sucessos no âmbito da saúde pública do século XX (SCHOENY, 2010), traduzido pela drástica redução do índice de mortalidade relacionado com doenças transmissíveis por via hídrica. Todavia, o surgimento recente de grandes surtos de doenças relacionadas com a qualidade da água - tais como: gastroenterite por $E$. coli na Alemanha (2011) e em Walkerton, Ontário, Canada (2000); criptosporidiose em Milwaukee, Wisconsin, USA (1993); cólera em Angola, Haiti, Cuba, República Dominicana (2013) - serviu como uma dramática chamada de atenção para a necessidade da aplicação e controlo persistentes dos processos de desinfeção. Esta sucessão de surtos constitui um sério alerta para a necessidade de uma contínua investigação e reavaliação das técnicas aplicadas, de modo a otimizar a sua eficácia e garantir uma água segura para abastecimento público.

Em Portugal, a desinfeção da água é agora obrigatória, constituindo um importante instrumento na mitigação do risco de disseminação de doenças infeciosas com origem hídrica. Os processos de desinfeção mais aplicados no tratamento de abastecimento utilizam como agentes desinfetantes os compostos clorados, o ozono e a radiação UV, com vantagens, inconvenientes e condicionalismos específicos resultantes das suas propriedades oxidantes e/ou desinfetantes (RODRIGUES, 2014). A escolha do agente deve assegurar um compromisso entre a eficácia (inativação de microrganismos), a potencial formação de subprodutos e a garantia de um teor residual ao longo do sistema de distribuição, razões pelas quais não é possível utilizar o o zono ou a radiação UV como únicos desinfetantes no tratamento de água.

$O$ agente desinfetante mundialmente mais utilizado é o cloro (cerca de $80 \%$ ), por ainda ser um dos mais vantajosos em análises de custobenefício (GOPAL et al., 2007) e apresentar, também, propriedades oxidantes. Durante décadas, aplicaram-se compostos clorados na desinfeção da água sem o conhecimento de potenciais malefícios para a saúde humana decorrentes da eventual formação de subprodutos da desinfeção (SPD), muitos dos quais com propriedades carcinogénicas e/ou mutagénicas (SHARMA et al., 2014; LEGAYet al., 2010), quando da presença na água de substâncias percursoras (e.g., matéria orgânica natural (MON), brometos).

Das 700 espécies de SPD já identificadas, as presentes em maiores proporções são os trihalometanos (THM), os ácidos haloacéticos (HAA) e os haloacetonitrilos (HAN) (KRASNER, 2009). As espécies e a concentração dos SPD formados dependem em muito das condições em que ocorre a desinfeção, nomeadamente: da concentração e natureza da MON, da dose de cloro, do tempo de contacto, da temperatura e do pH (NIKOLAOU et al., 2004). A taxa de formação de THM aumenta com o tempo de contacto e concentração de MON, duplica por cada incremento de $10^{\circ} \mathrm{C}$ na temperatura da água, triplica com o aumento de uma unidade no valor do $\mathrm{pH}$.

Em relação a este importante parâmetro, convém referir que, ao invés do que acontece com os THM e os ácidos haloacéticos (HAA), a diminuição do $\mathrm{pH}$ incrementa a formação de haloacetonitrilos (HAN) genotóxicos e de tricloraminas tóxicas e indutoras de asma, facto que é particularmente relevante em instalações de lazer, como as piscinas interiores (HANSEN et al,, 2012).

Os THM com presença mais frequente na água são 0 clorofórmio (TCM), o bromodiclorometano (BDCM), 0 dibromoclorometano (DBCM) e o bromofórmio (TBM), cuja soma constitui o parâmetro THM totais (THMt) objeto das normas de qualidade da água em vigor, adquirindo, por isso, uma particular relevância na vigilância sanitária dos sistemas de abastecimento de água. Nesse sentido, impõe-se a adoção de estratégias de minimização eficazes e sistemáticas baseadas em alterações no esquema de tratamento da água, nomeadamente na oxidação de percursores e na seleção e controlo da dose de desinfetante adicionada (VIEIRA; DUARTE, 2007). 
No contexto da análise do risco para a saúde, resultante da exposição a THM, os modelos matemáticos, pelo seu baixo custo, rapidez de aplicação e obtenção de resultados, constituem uma importante ferramenta de apoio para os gestores operacionais de sistemas de abastecimento de água (Figura 1).

\section{Figura 1 - Modelos matemáticos como ferramenta de avaliação do risco para a saúde}

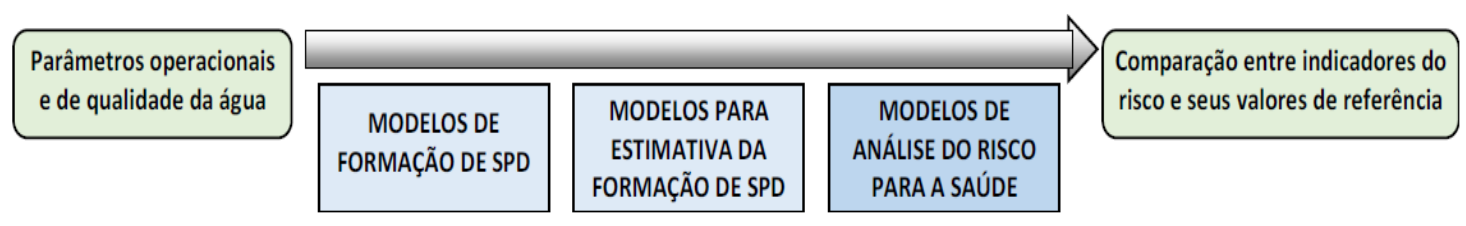

Os principais fatores a considerar na caracterização do risco associado à presença de SPD são a quantidade acumulada que é absorvida nos seres vivos, ao longo do tempo, e a sua toxicidade relativa. Durante o banho, podem ser transferidos para o corpo humano cerca do dobro dos THM ingeridos em $2 \mathrm{~L}$ de água (RICHARDSON et al., 2007).

Este aspeto é particularmente relevante no caso da exposição a THM em piscinas interiores, em que as vias da adsorção dérmica e da inalação assumem uma particular relevância, face ao tipo de utilização, tratamento e recirculação da água que conferem uma exposição (cumulativa) dos seus utentes (FLORENTIN et al., 2011; DUARTE, 2007).

Assim, um dos maiores desafios que se apresenta hoje no âmbito do abastecimento de água e das atividades de lazer é a otimização da relação entre a segurança microbiológica e 0 risco para a saúde associado à presença de THM e/ou bromatos originados nos processos de desinfeção mais utilizados no tratamento da água para consumo humano, objetivo do presente artigo.

\section{METODOLOGIA}

\subsection{Análise da ocorrência de THM na área de estudo}

O controlo e a monitorização da concentração de trihalometanos totais (THMt) na água distribuída em Portugal Continental têm vindo a ser realizado de forma positiva, não só porque a percentagem de análises realizadas (em relação às legalmente exigidas) supera os $99,6 \%$, mas, sobretudo, pelo facto de as concentrações de THMt detetadas, face ao valor paramétrico (VP) de referência, estarem em conformidade em mais de $99 \%$ das análises anuais realizadas no triénio 2011-2013 (ERSAR, 2012; 2013; 2015), embora se tenha verificado um ligeiro acréscimo de incumprimentos de 0,12 para 0,82\% nesse período. Com base nos boletins de análise publicados em Portugal, apresenta-se, na Figura 2, a evolução da concentração de THMt na torneira do consumidor nesse triénio, em termos de valores máximos e do percentil 95.

$\mathrm{Da}$ análise dos dados, constata-se uma tendência de aumento significativo dos valores máximos observados ao longo do triénio, embora os valores referentes ao percentil 95 tivessem apresentado uma tendência de crescimento muito ténue.

Numa observação global dos indicadores apresentados para cada entidade gestora de sistemas de abastecimento de água (SAA), constatou-se, de uma forma generalizada, que os resultados das análises da água na torneira do consumidor revelam um desempenho inferior ao verificado nos reservatórios.

Este facto está relacionado com a maior complexidade técnica na gestão das redes de distribuição de água e à existência de maiores vulnerabilidades desses sistemas, decorrentes quer das dificuldades no controlo operacional do processo de recloração no sistema de adução, quer do excessivo tempo de contacto água/cloro, nomeadamente em locais periféricos das redes de distribuição.

A área objeto deste estudo corresponde aos catorze municípios do distrito de Braga (Figura 3), que apresenta uma área de $2.673 \mathrm{~km}^{2}$ e uma população residente, em 2009, de 866 mil habitantes. 


\section{Figura 2 - Concentrações de THMt na água de abastecimento} em Portugal (triénio 2011-2013)

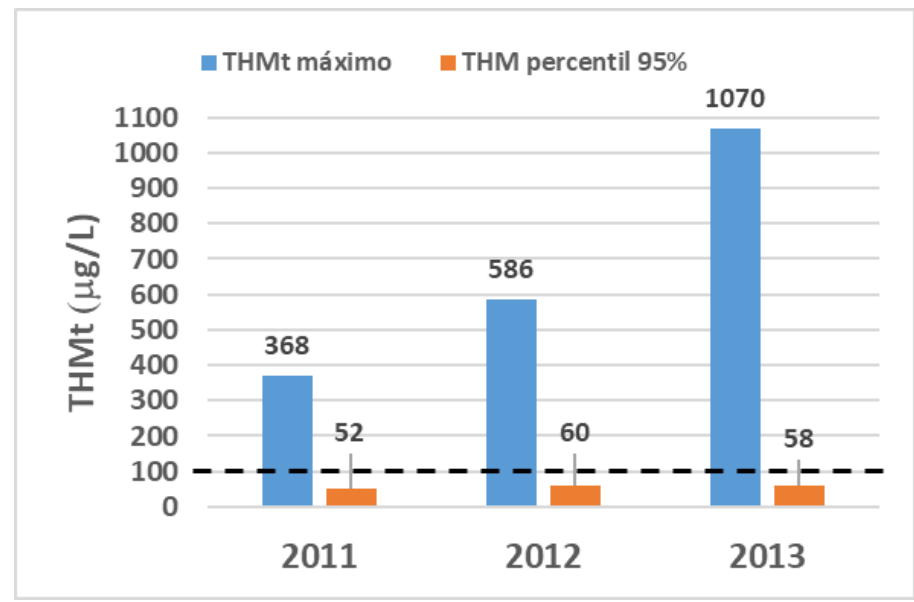

Fonte: ERSAR, 2012; 2013; 2015.

Figura 3 - Localização da área do estudo: concelhos do distrito de Braga (Portugal)

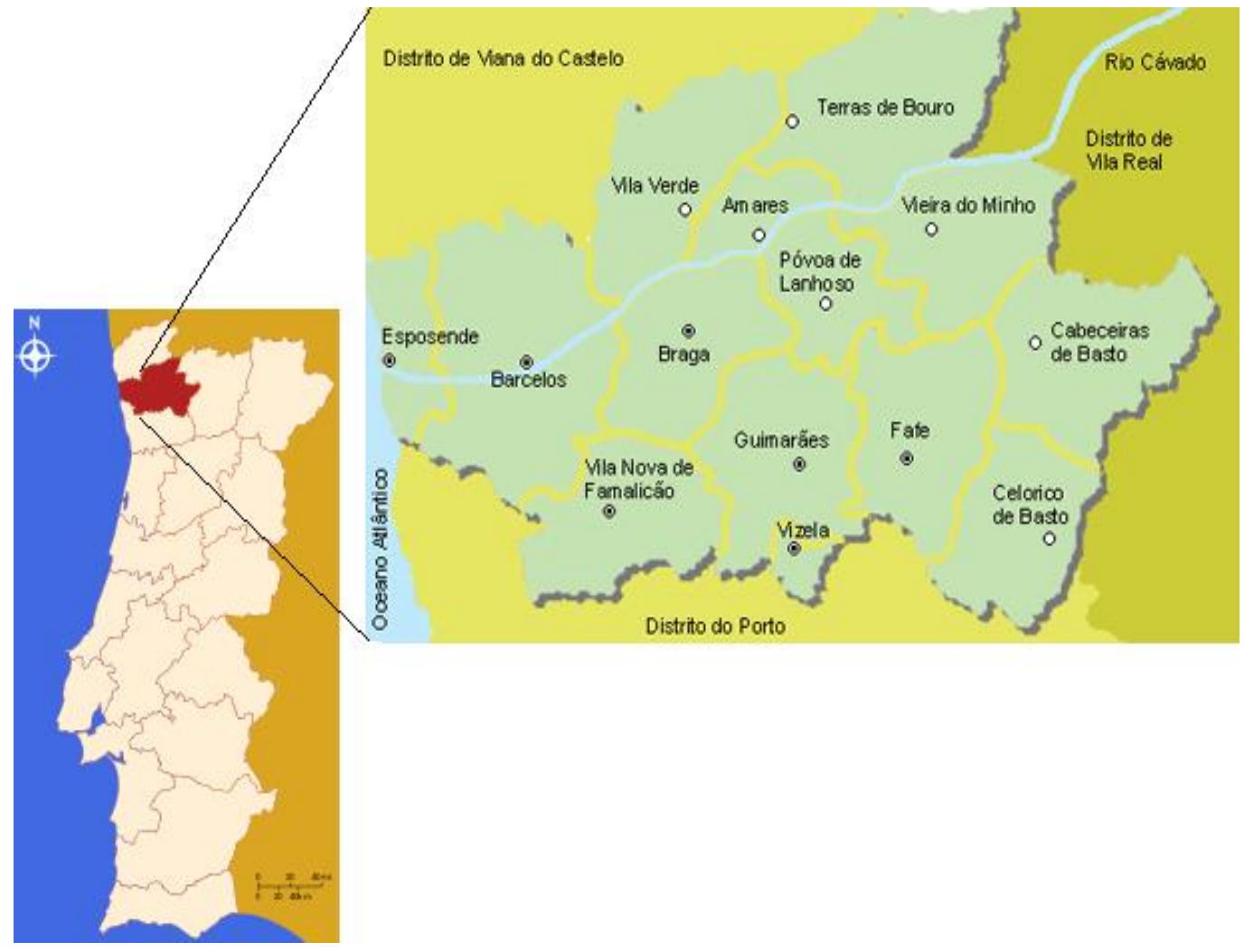

Fonte: Visitar Portugal.

Para a elaboração deste estudo, a análise dos dados relativos à qualidade da água monitorizada no utilizador final incidiu sobre um quinquénio, em que os valores relativos aos anos de 2009 e 2010 foram obtidos junto das entidades gestoras dos sistemas de abastecimento ou por consulta direta dos editais publicados trimestralmente pelas respetivas câmaras municipais (quando disponibilizados), e os referentes aos anos de 2011, 2012 e 2013 foram obtidos dos relatórios elaborados pela Entidade Reguladora do sector da água e dos resíduos em Portugal (ERSAR).

$\mathrm{Na}$ Tabela 1, apresenta-se uma síntese dos valores mínimos e máximos obtidos para a concentração de THM por consulta dos registos 
de análises (boletins de análise), referentes à água de abastecimento nos catorze municípios do distrito de Braga, que se encontram disponíveis, para consulta pública, nas páginas WEB de cada uma dessas municipalidades.

$\mathrm{Na}$ Figura 4, ilustra-se, como exemplo, o caso de Amares (população servida de 16.400 hab.), em que se registaram os valores máximos mais elevados (no quinquénio analisado) e situações de incumprimento face ao valor paramétrico $(\mathrm{VP}=100 \mu \mathrm{g} / \mathrm{L})$ definido na legislação em vigor, tendo como agravante uma clara tendência de subida nesse período. Os dados foram igualmente retirados dos boletins de análise publicados na página WEB do respetivo município.

Tabela 1 - Concentração de THMt na água de abastecimento dos municípios do distrito de Braga

\begin{tabular}{|c|c|c|c|c|c|c|}
\hline \multirow{2}{*}{\multicolumn{2}{|c|}{ Concelhos }} & \multicolumn{5}{|c|}{ Trihalometanos totais $(\mu \mathrm{g} / \mathrm{L})$} \\
\hline & & \multirow{2}{*}{$\frac{2009}{8}$} & \multirow{2}{*}{$\begin{array}{c}2010 \\
31\end{array}$} & \multirow{2}{*}{$\frac{2011}{7}$} & \multirow{2}{*}{$\frac{2012}{7}$} & \multirow{2}{*}{$\frac{2013}{7}$} \\
\hline \multirow{2}{*}{ Amares } & Min & & & & & \\
\hline & Max & 38 & 128 & 232 & 276 & 661 \\
\hline \multirow{2}{*}{ Barcelos } & Min & - & - & 0,5 & 20 & 20 \\
\hline & Max & - & - & 20 & 31,63 & 21,7 \\
\hline \multirow{2}{*}{ Braga } & Min & 111,5 & 10 & 18 & 7 & 18 \\
\hline & Max & 32,2 & 24 & 31 & 40,4 & 30 \\
\hline \multirow{2}{*}{ Cabeceiras de Basto } & Min & 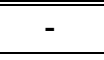 & - & 0,5 & 0,5 & 0,5 \\
\hline & Max & - & - & 21,4 & 58,5 & 94,2 \\
\hline \multirow{2}{*}{ Celorico de Basto } & Min & - & - & $3,3,5$ & 7 & $\overline{7}$ \\
\hline & Max & - & - & 34 & 25,5 & 76,4 \\
\hline \multirow{2}{*}{ Esposende } & Min & 10 & 7 & 14 & 111,9 & 25,5 \\
\hline & Max & 25 & 27 & 50,9 & 38,7 & 29,7 \\
\hline \multirow{2}{*}{ Fafe } & Min & 1 & 10 & 2 & 7 & 7 \\
\hline & Max & 42 & 49 & 93 & 69 & 41 \\
\hline \multirow{2}{*}{ Guimarães } & Min & 20,2 & "50,9 & $9,9,94$ & 3 & 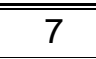 \\
\hline & Max & 40 & 67 & 132,27 & 52 & 47 \\
\hline \multirow{2}{*}{ Póvoa de Lanhoso } & Min & 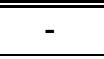 & 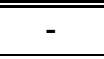 & 7 & 7 & $\overline{77}$ \\
\hline & Max & - & - & 56 & 117 & 94,5 \\
\hline \multirow{2}{*}{ Terras de Bouro } & Min & - & - & 7 & 7 & 7 \\
\hline & Max & - & - & 14 & 100 & 8,8 \\
\hline \multirow{2}{*}{ Vieira do Minho } & Min & - & - & $0,0,1$ & 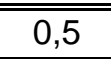 & $\overline{7}$ \\
\hline & Max & - & - & 2,6 & 7 & 7,4 \\
\hline \multirow{2}{*}{ V.N. de Famalicão } & Min & 9,3 & 8,4 & 6,9 & 5,83 & $1,1,57$ \\
\hline & Max & 34 & 16 & 24,17 & 26,3 & 13,43 \\
\hline \multirow{2}{*}{ Vila Verde } & Min & $1,1,5$ & 7 & 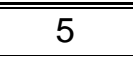 & 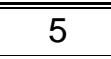 & 7 \\
\hline & Max & 57,7 & 119 & 195 & 162 & 98 \\
\hline \multirow{2}{*}{ Vizela } & Min & 20,2 & 50,9 & $9,9,94$ & 3 & 7 \\
\hline & Max & 40 & 67 & 132,27 & 52 & 47 \\
\hline
\end{tabular}




\section{Figura 4 - Evolução da concentração de THMt no município de Amares (2009-2013)}

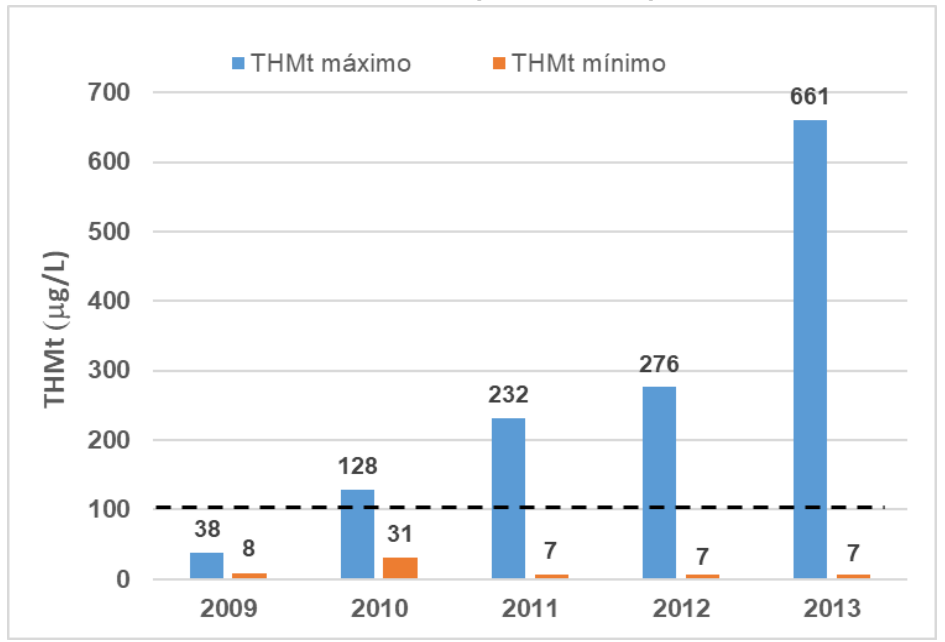

O valor máximo detetado de $661 \mu \mathrm{g} / \mathrm{L}$ constitui mesmo um dos mais elevados em relação aos que foram observados em Portugal, nesse quinquénio.

Neste trabalho, serão apenas apresentados os resultados da avaliação do risco, resultante da exposição a THMt, considerando um cenário de gestão gravoso, correspondente à ocorrência frequente, em cada um desses municípios, de concentrações de THMt semelhantes às dos valores máximos observados no quinquénio analisado. Embora este cenário não seja obviamente o mais expectável, ele pretende traduzir uma situação futura extrema (plausível) baseada em situações já ocorridas.

\subsection{Análise do risco da presença de trihalometanos}

A análise do risco para a saúde é, por definição, um processo que visa estimar a natureza e probabilidade de ocorrência de efeitos adversos na saúde em humanos expostos a determinados compostos químicos e/ou agentes biológicos, no presente e no futuro.

A avaliação e caracterização dos perfis de exposição são fases fundamentais no processo de análise do risco, sendo necessário considerar, neste caso, todos os usos da água que constituam vias de exposição. Assim, nos últimos anos, têm vindo a ser efetuados estudos de avaliação do risco de contrair cancro ao longo da vida (Lifetime Cancer Risk - CR), considerando as múltiplas vias de exposição à presença de THM: ingestão, inalação e absorção dérmica
(PAOPUREE et al., 2010; BASU et al., 2011; PENTAMWA et al., 2013; AMJAB et al., 2013).

A caracterização do risco associado à exposição a THM traduz-se, usualmente, por um indicador dimensional (CR) que representa a quantificação da probabilidade de se contrair cancro ao longo da vida. Ou seja, um valor CR de 1 para 1 milhão (1E-06) traduz a probabilidade de uma única pessoa ter cancro no universo de um milhão de pessoas, igualmente expostas e de modo permanente ao agente contaminante durante a sua vida.

Além do risco associado ao efeito carcinogénico dos THM, procedeu-se também à avaliação do risco referente à componente nãocarcinogénica. O indicador HI (Hazard Index) é um indicador adimensional usado na avaliação de outros efeitos adversos para a saúde (efeito sobre um organismo ou órgão específico) provocados por um ou mais contaminantes (e.g., distúrbios comportamentais no nível do sistema nervoso central, problemas de desenvolvimento intrauterino, alterações hepáticas).

$\mathrm{O} \mathrm{HI}$ representa a relação entre uma estimativa da exposição diária ao contaminante em causa e uma dose de referência crónica $(\mathrm{RfD})$. Este parâmetro RfD representa $\circ$ valor limite de assimilação diária da substância que é atualmente reconhecido pela comunidade científica como sendo de efeito nulo. Desta forma, uma magnitude do indicador $\mathrm{HI}$, no seu resultado final (acumulado dos vários contaminantes e vias de exposição), igual ou inferior a 1 , indicia a probabilidade de não ocorrerem efeitos adversos na saúde. É de salientar, no entanto, que, devido 
ao conservadorismo e incerteza associada à determinação da RfD, um HI final superior a 1 não implica necessariamente uma efetiva deterioração futura da condição biológica dos indivíduos (IRIS, 2014).

Em ambos os indicadores (CR e HI), o espaço temporal considerado é a esperança de vida, por ser já reconhecido que a ocorrência de cancro está associada a uma exposição de longo prazo e não a eventos episódicos.

\subsection{Método de cálculo dos indicadores (CR e HI)}

Neste estudo, o método de cálculo do CR associado aos THM baseou-se nos programas IRIS (Integrated Risk Information System) e RAIS (Risk Assessment Information System) (IRIS, 2014; RAIS, 2014; USEPA, 2011), desenvolvidos para avaliação e proteção da saúde humana, os quais integram bases de dados alargadas de informação relativas ao risco associado ao efeito resultante da exposição a determinadas substâncias químicas.
O indicador risco de cancro (CR) é obtido multiplicando o parâmetro correspondente à exposição diária crónica (CDI) por um fator potência (PF) atribuído a cada substância, e cujos valores adotados neste estudo constam na Tabela 2.

O fator de potência representa uma estimativa plausível do limite superior da probabilidade de ocorrência de um determinado efeito resultante da assimilação de um composto químico ao longo da vida, ou seja, corresponde a um valor de toxicidade que define quantitativamente a relação estabelecida entre uma determinada dose absorvida (CDI) e a resposta do organismo humano.

A CDI total resultou da soma dos efeitos das três vias de exposição consideradas: ingestão, exposição dérmica e inalação, em que cada parcela é calculada por meio das equações seguintes (1 a 3), em que cada uma das variáveis está identificada na Tabela 3.

Tabela 2 - Fator de potência dos trihalometanos para cálculo do indicador risco de cancro (CR)

\begin{tabular}{|c|c|c|c|}
\hline \multirow[b]{2}{*}{ Trihalometano } & \multicolumn{3}{|c|}{ Fator de Potência (PF) $[(\mathrm{mg} / \mathrm{kg} / \mathrm{dia})]^{*}$} \\
\hline & $\begin{array}{c}\text { Ingestão } \\
\text { (IRIS, 2014) }\end{array}$ & $\begin{array}{c}\text { Absorção Dérmica } \\
\text { (RAIS, 2014) }\end{array}$ & $\begin{array}{c}\text { Inalação } \\
(\text { IRIS, 2014) }\end{array}$ \\
\hline Clorofórmio $\left(\mathrm{CHCl}_{3}\right)$ & 0,0061 & 0,0305 & 0,081 \\
\hline Diclorobromometano $\left(\mathrm{CHCl}_{2} \mathrm{Br}\right)$ & 0,062 & 0,0633 & $0,13^{* *}$ \\
\hline Clorodibromometano $\left(\mathrm{CHClBr}_{2}\right)$ & 0,084 & 0,14 & $0,094^{* *}$ \\
\hline Bromofórmio $\left(\mathrm{CHBr}_{3}\right)$ & 0,0079 & 0,0132 & 0,0039 \\
\hline
\end{tabular}

Fonte: IRIS, 2014; Lee et al., 2013.

$C D I_{I N G E S T \tilde{O} O}=\frac{C W \times I R \times E F \times E D \times C F}{B W \times A T}$

$C D I_{A B S O R C \hat{A} O \text { DÉRMICA }}=\frac{C W \times S A \times F \times P C \times E T \times E F \times E D \times C F}{B W \times A T}$

$C D I_{I N A L A C ̧ \tilde{A} O}=\frac{C a i r \times V R \times A E \times E T \times E F \times E D \times C F}{B W \times A T}$ 
No caso da inalação, admitiu-se que a exposição durante o duche foi a determinante em detrimento da que ocorre durante a preparação de alimentos. A modelação da exposição por esta via foi desenvolvida com base na teoria proposta por Little (1992), que permite estimar os THM volatilizados nas instalações sanitárias.

O valor do $\mathrm{CR}$ global $\left(\mathrm{CR}_{\mathrm{T}}\right)$ resulta do efeito cumulativo associado aos riscos referentes a cada THM decorrentes das três diferentes vias de exposição referidas, sendo calculado pela equação (4).

$$
\mathrm{CR}_{\mathrm{T}}=\sum \mathrm{CR}_{\mathrm{i}, \mathrm{j}}
$$

em que: $i$ corresponde a cada um dos 4THM considerados e $j$ a cada uma das 3 vias de exposição.

A estimativa do indicador do risco referente aos efeitos não-carcinogénicos dos THM $(\mathrm{HI})$ foi efetuada dividindo o fator de exposição diária crónica (CDI) com a dose de referência crónica ( $R f D$ ), sendo consideradas, neste processo, as vias de exposição por ingestão oral e assimilação dérmica para cada um dos quatro THM analisados.

A Tabela 3 apresenta, além da identificação das variáveis, uma síntese dos valores desses diferentes parâmetros de entrada requeridos pelo modelo e usados no cálculo desses indicadores (RODRIGUES, 2014).

Face à enorme variabilidade dos valores bibliográficos relativos a cada um destes parâmetros, houve a preocupação, neste estudo, de adaptar, sempre que possível, os valores típicos das diversas variáveis do modelo à realidade da população portuguesa (e.g., esperança média de vida e massa corporal), especificando-se valores diferentes para as variáveis relativas a homens e a mulheres, pelo que os indicadores do risco refletem necessariamente essa distinção. Considerou-se, então, que a esperança média de vida em Portugal, relativa a homens e a mulheres, é de 76,9 e 82,8 anos, respetivamente, em 2013. A massa corporal (BW) adotada foi de $74 \mathrm{~kg}$ para os homens e $64 \mathrm{~kg}$ para as mulheres. A caracterização mais detalhada dos eventos de exposição considerados pode ser consultada em Rodrigues (2014).

A aplicação deste método de avaliação do risco requer, como referido, os valores de concentrações referentes a cada um dos quatro THM. Dado que nem todas as análises da qualidade de água disponibilizadas apresentavam os valores de THM de forma desagregada, procedeu-se ao tratamento estatístico dos dados válidos recolhidos, de forma a determinar o peso de cada THM na quantidade de THM totais, no nível regional e nacional.

De modo a implementar o cálculo automático destes indicadores, neste estudo, foi desenvolvido um modelo, designado por CRacel (RODRIGUES, 2014), no formato de folha de cálculo usando o programa MS Excel, dado ser uma ferramenta informática de utilização universal.

A validação do modelo foi realizada por intermédio da introdução dos parâmetros de entrada considerados no trabalho realizado por Amjad (2013) e posterior comparação com os respetivos resultados finais.

A título ilustrativo, apresenta-se na Figura 5 a estrutura da folha de entrada de dados deste modelo, com os valores adotados no estudo realizado.

A análise de sensibilidade constitui uma ferramenta que visa determinar a influência que uma variação imposta num dado parâmetro de entrada representa no resultado final fornecido por um modelo, de modo a identificar as variáveis críticas, sendo realizada impondo a flutuação percentual dos valores adotados e observando os efeitos subsequentes nos resultados obtidos.

Neste estudo, a análise de sensibilidade incidiu sobre os parâmetros referentes ao volume de água ingerido, tempo de exposição à água, taxa de ventilação do quarto de banho, caudal fornecido pelo chuveiro e concentrações dos quatro THM considerados. A variação imposta nessa análise foi de $\pm 25 \%$ sobre o valor adotado destas variáveis, utilizando-se o módulo de teste de hipóteses integrado no modelo CRacel.

\section{RESULTADOS E DISCUSSÃO}

O resultado da análise estatística dos dados de THM recolhidos, para o distrito de Braga, permitiu estabelecer uma proporção (média) entre os vários tipos de THM presentes na água de abastecimento (Figura 6). 
Tabela 3 - Síntese dos parâmetros de entrada usados no cálculo dos indicadores do risco

\begin{tabular}{|c|c|c|c|c|}
\hline $\begin{array}{c}\text { Tipo de } \\
\text { Parâmetro }\end{array}$ & Parâmetro & Notação & Unidade & Valor \\
\hline \multirow[t]{7}{*}{ Geral } & $\begin{array}{c}\text { Concentração de THM na } \\
\text { água }\end{array}$ & $\mathrm{Cw}$ & $\mu \mathrm{g} / \mathrm{L}$ & Tabela 2 \\
\hline & Esperança média de vida & AT & dias & $\begin{array}{l}\text { Homens:76.91*365 } \\
\text { Mulheres:82.79*365 }\end{array}$ \\
\hline & Massa corporal & BW & $\mathrm{Kg}$ & $\begin{array}{l}\text { Homens: } 74 \\
\text { Mulheres: } 64\end{array}$ \\
\hline & Fator de conversão & $\mathrm{CF}$ & $\mathrm{L} / \mathrm{cm}^{3}$ & 0.001 \\
\hline & Duração exposição & ED & anos & $\begin{array}{l}\text { Homens:76.91 } \\
\text { Mulheres:82.79 }\end{array}$ \\
\hline & Frequência exposição & $\mathrm{EF}$ & dias/ano & 365 \\
\hline & Tempo exposição & ET & $\min /$ dia & 35 \\
\hline Ingestão & Taxa de ingestão & IR & $\mathrm{L} / \mathrm{dia}$ & 2 \\
\hline \multirow[t]{3}{*}{$\begin{array}{l}\text { Absorção } \\
\text { Dérmica }\end{array}$} & Área contacto pele & SA & $\mathrm{m}^{2}$ & $\begin{array}{c}(4 \mathrm{BW}+7) /(\mathrm{BW}+90) \\
\text { Homens: } 1.85 \\
\text { Mulheres: } 1.71\end{array}$ \\
\hline & $\begin{array}{l}\text { Proporção pele em contacto } \\
\text { com a água }\end{array}$ & $\mathrm{F}$ & $\%$ & 90 \\
\hline & Coeficiente permeabilidade & $\mathrm{PC}$ & $\mathrm{cm} / \mathrm{h}$ & $\begin{array}{c}0.00683 \text { (Clorofórmio) } \\
0.00402\left(\mathrm{BDCM}^{\mathrm{a}}\right) \\
0.00289\left(\mathrm{DBCM}^{\mathrm{b}}\right) \\
0.0026(\text { Bromofórmio })\end{array}$ \\
\hline \multirow[t]{9}{*}{ Inalação } & Concentração de THM no ar & Cair & $\mathrm{mg} / \mathrm{L}$ & modelo de Little \\
\hline & Taxa respiratória & VR & $\mathrm{m}^{3} / \mathrm{h}$ & $\begin{array}{l}\text { Homens:0.84 } \\
\text { Mulheres:0.66 }\end{array}$ \\
\hline & $\begin{array}{l}\text { Eficiência de absorção } \\
\text { alveolar }\end{array}$ & $\mathrm{AE}$ & $\%$ & 50 \\
\hline & Volume quarto banho & $\mathrm{V}_{\mathrm{S}}$ & $\mathrm{m}^{3}$ & 5 \\
\hline & Caudal de água do duche & $\mathrm{Q}_{\mathrm{L}}$ & $\mathrm{L} / \mathrm{min}$ & 5 \\
\hline & Caudal de ventilação & $\mathrm{Q}_{\mathrm{G}}$ & $\mathrm{L} / \mathrm{min}$ & 50 \\
\hline & Temperatura da água & $\mathrm{T}$ & ${ }^{\circ} \mathrm{C}$ & 40 \\
\hline & $\begin{array}{c}\text { Constante adimensional de } \\
\text { Henry a } 40^{\circ} \mathrm{C}\end{array}$ & $\mathrm{H}$ & & $\begin{array}{c}0.25 \text { (Clorofórmio) } \\
0.124\left(\mathrm{BDCM}^{\mathrm{a}}\right) \\
0.0526\left(\mathrm{DBCM}^{\mathrm{b}}\right) \\
0.0501(\text { Bromofórmio })\end{array}$ \\
\hline & $\begin{array}{l}\text { Coeficiente de transferência } \\
\text { de massa }\end{array}$ & $\mathrm{K}_{\mathrm{OL}} \mathrm{A}$ & $\mathrm{L} / \mathrm{min}$ & $\begin{array}{c}7.4 \text { (Clorofórmio) } \\
5.9\left(\mathrm{BDCM}^{\mathrm{a}}\right) \\
4.6\left(\mathrm{DBCM}^{\mathrm{b}}\right) \\
3.7(\text { Bromofórmio })\end{array}$ \\
\hline $\begin{array}{c}\text { HI } \\
\text { (Hazard } \\
\text { Index })\end{array}$ & Dose referência & RfD & $\mathrm{mg} / \mathrm{Kg} / \mathrm{dia}$ & $\begin{array}{c}0.01 \text { (Clorofórmio) } \\
0.02\left(\mathrm{BDCM}^{\mathrm{a}}\right) \\
0.02\left(\mathrm{DBCM}^{\mathrm{b}}\right) \\
0.02(\text { Bromofórmio })\end{array}$ \\
\hline
\end{tabular}

\footnotetext{
${ }^{a}$ Diclorobromometano

${ }^{\mathrm{b}}$ Dibromoclorometano
} 
Figura 5 - Estrutura dos parâmetros de entrada no modelo CRacel

\begin{tabular}{|c|c|c|c|c|}
\hline \multicolumn{5}{|c|}{ Cancer Risk } \\
\hline \multicolumn{5}{|c|}{ Concentrações máximas diferenciadas de trihalometanos ( $\mu \mathrm{g} / \mathrm{L}$ ) } \\
\hline Concelhos/Casos em Estudo & $\mathrm{CHCl}_{3}$ & $\mathrm{CHBr}_{3}$ & $\mathrm{CHCl}_{2} \mathrm{Br}$ & $\mathrm{CHBr}_{2} \mathrm{Cl}$ \\
\hline Amares & 587 & 52 & 25 & 22 \\
\hline Barcelos & 11 & 20 & 6,5 & 4,2 \\
\hline Braga* & 9 & 9,3 & 6,6 & 5,1 \\
\hline Cabeceiras de Basto & 0,5 & 1,2 & 4,6 & 4,3 \\
\hline Celorico de Basto & 14 & 40 & 8,7 & 18 \\
\hline Esposende & 17 & 1,2 & 7,8 & 6,3 \\
\hline Fafe* & 12,3 & 12,71 & 9,02 & 6,97 \\
\hline Guimarães & 19 & 7 & 35 & 8 \\
\hline Póvoa de Lanhoso & 27 & 56 & 11 & 27 \\
\hline Terras de Bouro* & 2,64 & 2,73 & 1,93 & 1,5 \\
\hline Vieira do Minho & 7 & 7 & 7 & 7 \\
\hline V.N. de Famalicão & 6,1 & 0,53 & 4,1 & 2,7 \\
\hline Vila Verde & 43 & 32 & 10 & 11 \\
\hline Vizela & 19 & 7 & 35 & 8 \\
\hline \multicolumn{5}{|c|}{ Factores Potência PF (mg/kg.dia) ${ }^{-1}$} \\
\hline Via Exposição & $\mathrm{CHCl}_{3}$ & $\mathrm{CHBr}_{3}$ & $\mathrm{CHCl}_{2} \mathrm{Br}$ & $\mathrm{CHBr}_{2} \mathrm{Cl}$ \\
\hline Ingestão & 0,0061 & 0,0079 & 0,062 & 0,084 \\
\hline Abs.Dérmica & 0,0305 & 0,0132 & 0,0633 & 0,14 \\
\hline Inalação & 0,081 & 0,0039 & 0,13 & 0,094 \\
\hline Parâmetro & $\mathrm{CHCl}_{3}$ & $\mathrm{CHBr}_{3}$ & $\mathrm{CHCl}_{2} \mathrm{Br}$ & $\mathrm{CHBr}_{2} \mathrm{Cl}$ \\
\hline H- Const. Henry $40^{\circ} \mathrm{C}$ & 0,25 & 0,0501 & 0,124 & 0,0526 \\
\hline $\begin{array}{l}\text { PC - Coefi. permeabilidade } \\
(\mathrm{cm} / \mathrm{h})\end{array}$ & 0,00683 & 0,0026 & 0,00402 & 0,00289 \\
\hline $\begin{array}{c}\mathbf{K}_{\mathrm{ol}} \mathbf{A} \text { - Coef. transferência } \\
\text { massa }(\mathrm{L} / \mathrm{min})\end{array}$ & 7,4 & 3,7 & 5,9 & 4,6 \\
\hline $\begin{array}{c}\text { RfD - Dose referência } \\
(\mathrm{mg} / \mathrm{Kg} / \mathrm{dia})\end{array}$ & 0,01 & 0,02 & 0,02 & 0,02 \\
\hline
\end{tabular}

Variáveis do modelo

\begin{tabular}{|c|c|c|c|c|}
\hline \multicolumn{5}{|c|}{ Variáveis do modelo } \\
\hline \begin{tabular}{|c|} 
Tipo \\
Parâmetro
\end{tabular} & Parâmetro & Var. & Unidade & Valor \\
\hline \multirow{9}{*}{ Geral } & Esperança média de vida -Homens & \multirow{2}{*}{ AT } & \multirow{2}{*}{ dia } & 28072 \\
\hline & Esperança média de vida - Mulheres & & & 30218 \\
\hline & Massa corporal - Homens & \multirow{2}{*}{ BW } & \multirow{2}{*}{$\mathrm{Kg}$} & 74 \\
\hline & Massa corporal - Mulheres & & & 64 \\
\hline & Fator de conversão & $\mathrm{CF}$ & $\mathrm{L} / \mathrm{cm}^{3}$ & 0,001 \\
\hline & Duração da exposição - Homens & \multirow{2}{*}{ ED } & \multirow{2}{*}{ ano } & 76,91 \\
\hline & Duração da exposição - Mulheres & & & 82,79 \\
\hline & Frequência de exposição & $\mathrm{EF}$ & dia/ano & 365 \\
\hline & Tempo de exposição & ET & $\mathrm{min} / \mathrm{dia}$ & 35 \\
\hline \begin{tabular}{|l|} 
Ingestão \\
\end{tabular} & Taxa de ingestão & $\mathrm{IR}$ & L/dia & 2 \\
\hline \multirow{3}{*}{$\begin{array}{c}\text { Absorção } \\
\text { Dérmica }\end{array}$} & Área de contacto da pele - Homens & \multirow{2}{*}{ SA } & \multirow{2}{*}{$\mathrm{m}^{2}$} & 1,85 \\
\hline & Área de contacto da pele - Mulheres & & & 1,71 \\
\hline & Proporção da pele em contacto com água & $\mathrm{F}$ & 0a 1 & 0,9 \\
\hline \multirow{7}{*}{ Inalação } & Taxa respiratória - Homens & \multirow{2}{*}{ VR } & \multirow{2}{*}{$\mathrm{m} 3 / \mathrm{h}$} & 0,84 \\
\hline & Taxa respiratória - Mulheres & & & 0,66 \\
\hline & Eficiência de absorção alveolar & $\mathrm{AE}$ & 0 a 1 & 0,5 \\
\hline & Volume quarto de banho & $\mathrm{V}_{\mathrm{S}}$ & $\mathrm{m}^{3}$ & 5 \\
\hline & Caudal de água duche & $Q_{L}$ & $\mathrm{~L} / \mathrm{min}$ & 5 \\
\hline & Caudal de ventilação & $\mathrm{Q}_{\mathrm{G}}$ & $\mathrm{L} / \mathrm{min}$ & 50 \\
\hline & Temperatura da água & $T$ & $\stackrel{\circ}{ } \mathrm{C}$ & 40 \\
\hline
\end{tabular}

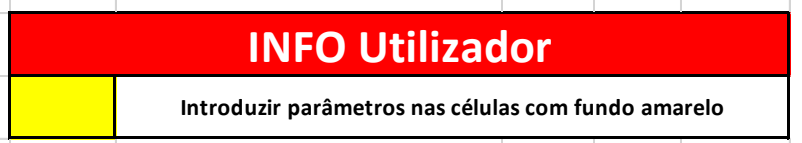

\title{
Figura 6 - Proporção entre os THM presentes na água de abastecimento do distrito de Braga
}

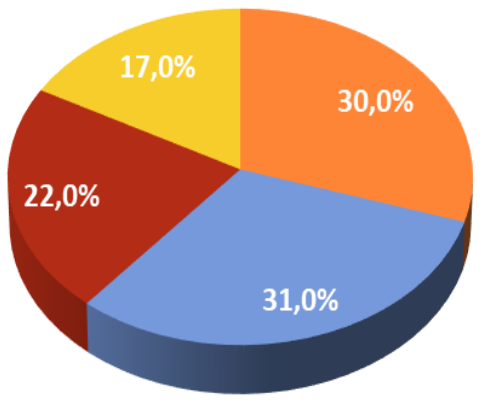

\author{
Clorofórmio \\ Bromofórmio \\ - Diclorobromometano \\ Dibromoclorometano
}

O resultado obtido permitiu constar que, contrariamente ao expectável, o bromofórmio foi o THM detetado com maior concentração, devido à presença frequente de iões brometo nas águas brutas, nesse distrito, onde as origens de água são maioritariamente superficiais e a constituição geológica das bacias hidrográficas é predominantemente de natureza granítica. Isto porque é frequente encontrar-se referências bibliográficas em que o clorofórmio surge como a forma de THM predominante, podendo atingir cerca de $60-70 \%$ do total.

Apresentam-se, em seguida, alguns dos resultados obtidos na pesquisa desenvolvida, selecionando-se os relativos à avaliação dos riscos globais, tanto para os carcinogénicos (indicador $\mathrm{CRT}$ ) como para os não-carcinogénicos (indicador $\mathrm{HI}$ ), de modo a permitir uma visão cumulativa dos efeitos relativos ao cenário de risco analisado neste trabalho, que corresponde 
ao mais gravoso dos definidos no estudo desenvolvido.

\subsection{Risco de cancro associado à exposição por múltiplas vias}

Na Figura 7, apresenta-se uma síntese dos resultados obtidos em cada município para 0 indicador do risco de cancro total (CRT), resultante dos efeitos cumulativos das várias vias de exposição aos THM (ingestão, absorção dérmica, inalação), em homens e mulheres, sendo aqui $o$ fator diferenciador a maior esperança de vida, no caso das mulheres, que implica uma duração de exposição superior ao longo da vida.

\section{Figura 7 - Valor total do indicador CR resultante das múltiplas vias de exposição aos THM, nos municípios do distrito de Braga}

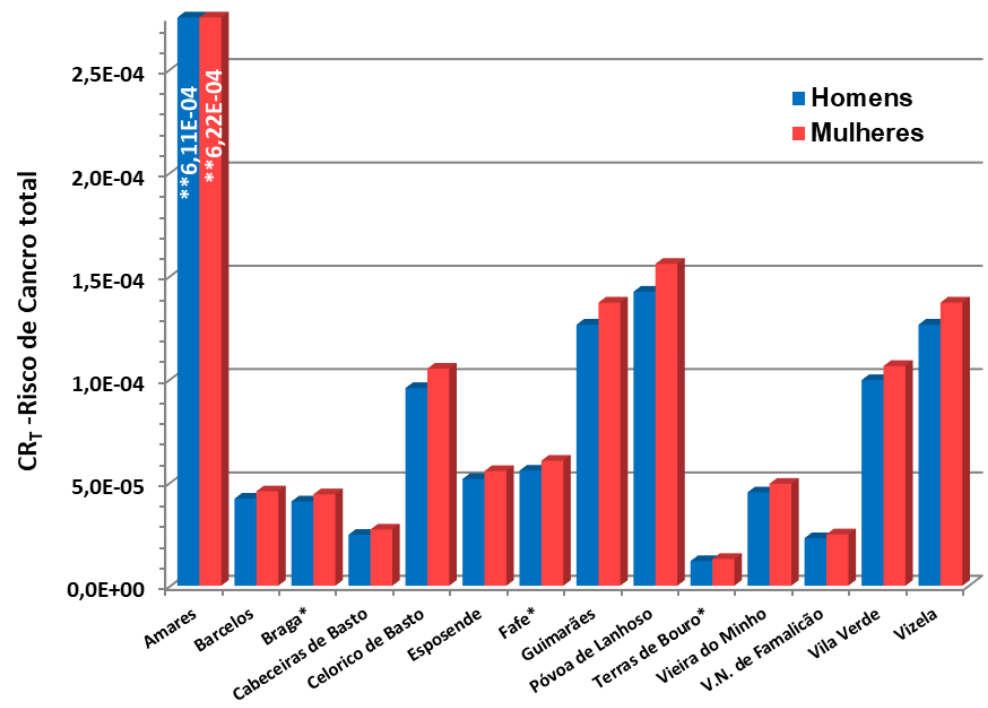

Os resultados obtidos revelam que nenhum dos municípios apresenta um risco inferior ao valor de referência. O município de Amares obteve o CR mais elevado, representando um risco 600 vezes superior ao limite do risco aceitável estipulado pela USEPA (2011).

Saliente-se, de novo, que os valores de concentração de THM considerados foram os máximos observados no quinquénio em análise e, por isso, representativos do mais elevado grau de risco, sendo um cenário extremo (plausível) mas não representativo das atuais condições reais.

A Figura 8 apresenta uma síntese do peso relativo de cada uma das vias de exposição no valor total do indicador risco de cancro (CRT), de modo a evidenciar ser a ingestão a via preponderante na quase totalidade dos municípios analisados, como seria expectável.

$\mathrm{Da}$ análise dos resultados, verifica-se que, em treze dos municípios analisados, a ingestão é a via preponderante no valor do risco de cancro total, seguida da inalação e, por fim, a de absorção dérmica.
Em Amares, o fator relativo à exposição por inalação é o mais relevante e não a ingestão, o que pode ser explicado pelo facto de ter sido aqui detetada uma concentração de clorofórmio bastante mais elevada do que nos outros municípios.

Dado que o clorofórmio é o THM que volatiliza com maior facilidade (ponto de ebulição mais baixo do que o dos outros), isso implica que existe uma maior exposição durante os duches e banhos, incrementando o peso relativo da via por inalação.

O cálculo do indicador HI é complementar ao CR e necessário como forma de avaliar os efeitos adversos não carcinogénicos decorrentes da exposição aos THM por ingestão e por absorção dérmica.

Na Figura 9, apresenta-se uma síntese dos resultados obtidos em cada município, para o indicador do risco não carcinogénico $\left(\mathrm{HI}_{\mathrm{T}}\right)$ resultante dos efeitos cumulativos destas duas vias de exposição, em homens e mulheres. 
Figura 8 - Peso relativo de cada via de exposição no valor do risco de cancro em cada município

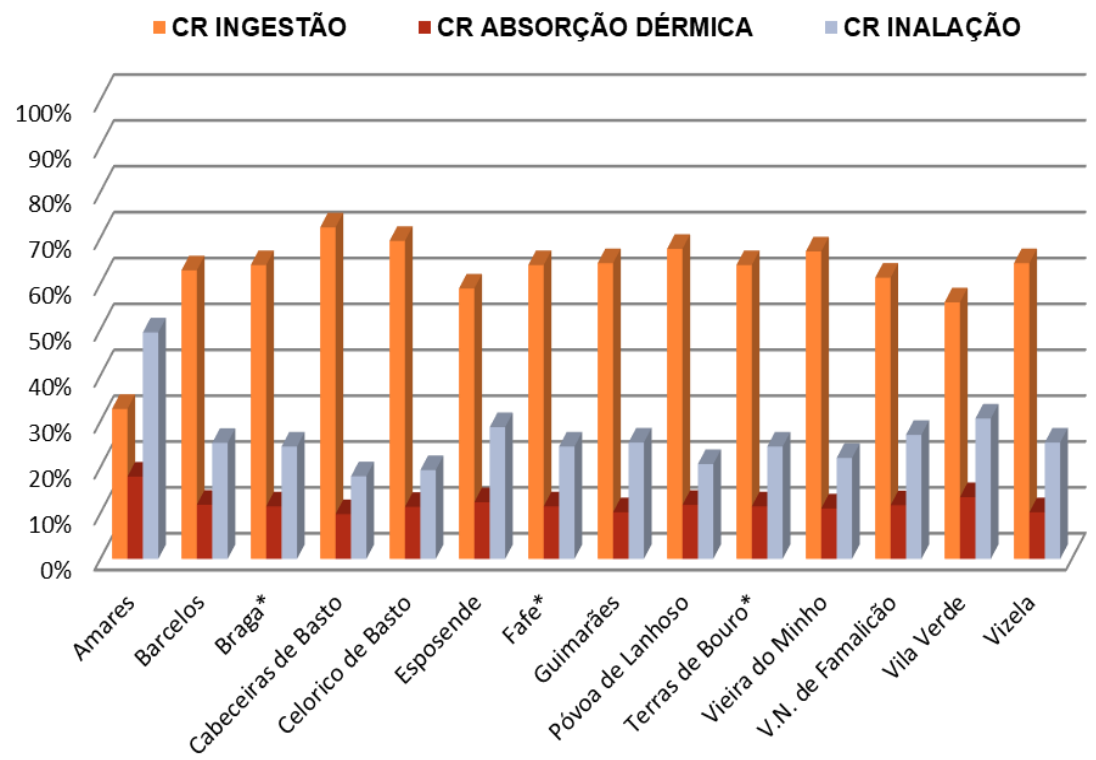

Figura 9 - Indicador de risco não cancerígeno $\left(\mathrm{HI}_{\mathrm{T}}\right)$ associado às múltiplas vias de exposição

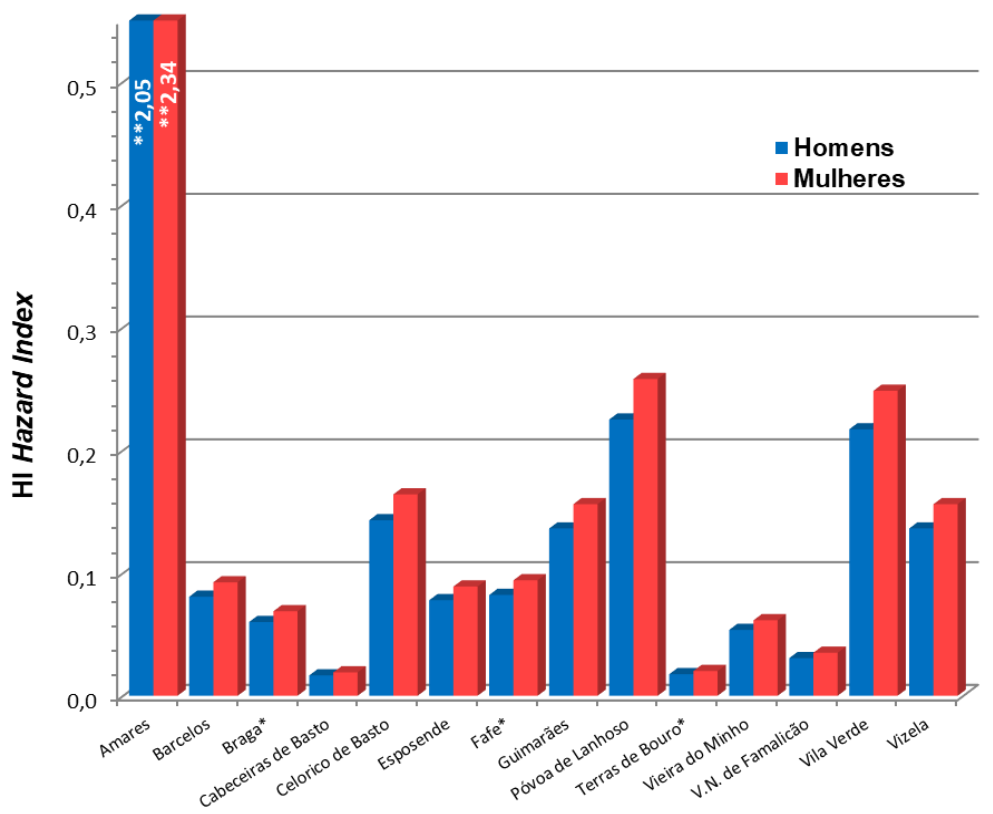

Para o município de Amares, obteve-se o valor mais elevado do $\mathrm{HI}$, sendo mesmo superior à unidade, pelo que é plausível que possam ocorrer aí efeitos adversos na saúde da população. Em todos os outros municípios deste distrito, os valores de HI são bastantes inferiores à unidade, traduzindo um risco não carcinogénico negligenciável.

\subsection{Análise de sensibilidade}

A Figura 10 apresenta, graficamente, os resultados da análise de sensibilidade, efetuada nas condições anteriormente descritas no item relativo à metodologia. 
Figura 10 - Análise de sensibilidade aos parâmetros de cálculo do indicador $\mathbf{C R}_{\mathbf{T}}$

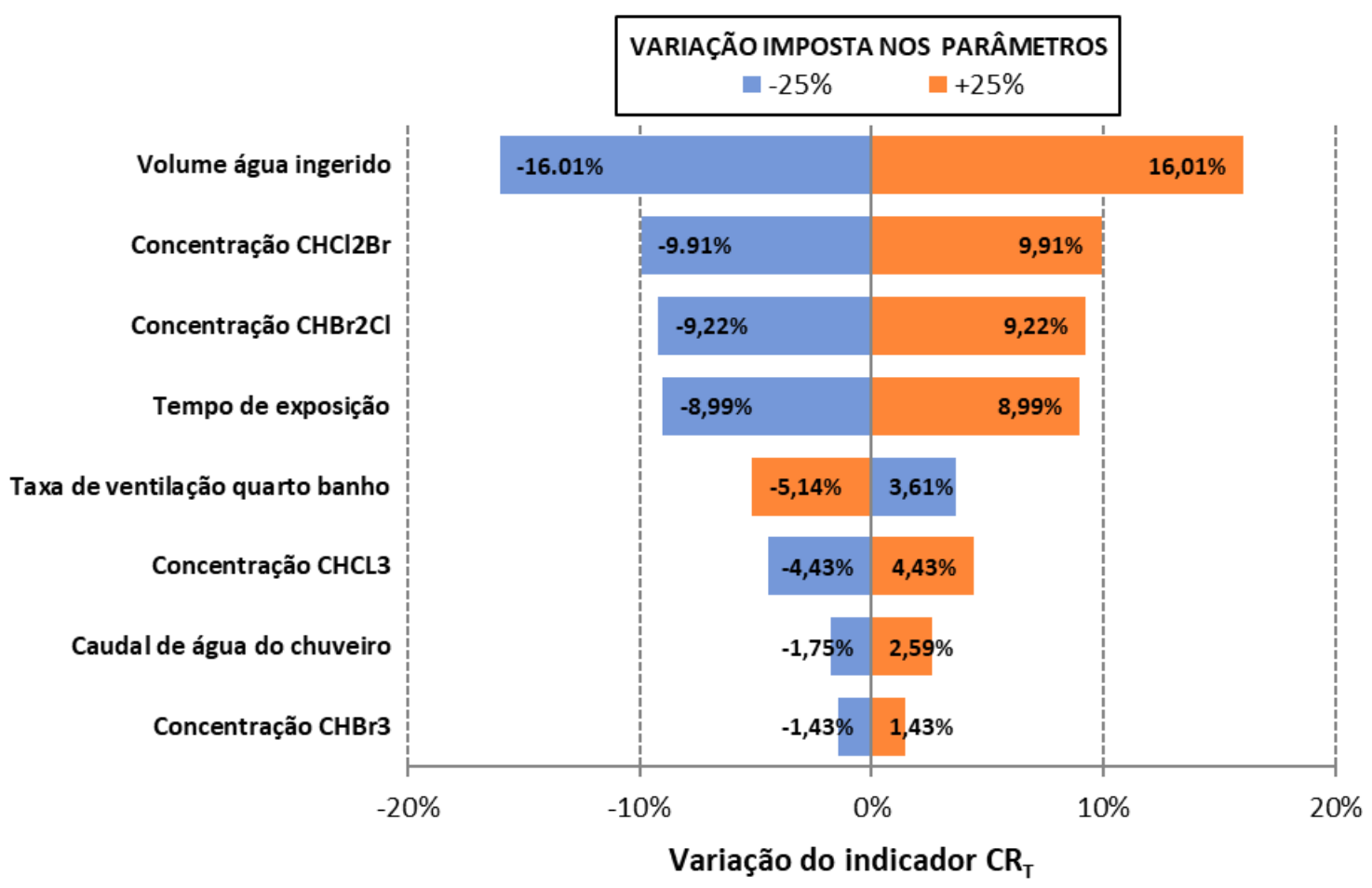

Os resultados obtidos nesta análise indiciam que o volume de água ingerido diariamente é a variável do modelo com mais impacto $(16 \%)$ no valor estimado para o indicador risco de cancro $\left(\mathrm{CR}_{\mathrm{T}}\right)$, seguido das variáveis tempo de exposição e concentrações de diclorobromometano e de dibromoclorometano, todas elas com um impacto no valor de $\mathrm{CR}_{\mathrm{T}}$ na ordem dos $9-10 \%$. Este facto dá relevância à necessidade de obter os valores de THM de forma desagregada e não como valor total (THMt).

Os restantes parâmetros induzem uma variação deste indicador bastante mais reduzida, pelo que a sua relevância e efeito na fiabilidade dos resultados fornecidos pelo modelo de análise do risco utilizado afiguram-se muito diminutos.

As tendências apresentadas na análise de sensibilidade foram idênticas para homens e mulheres em todos os municípios analisados neste estudo.

Com base nestes resultados, será possível aumentar a fiabilidade do método de avaliação do risco, com o aprofundamento do estudo e caracterização das variáveis identificadas como sendo as mais críticas em termos de efeito nos valores dos indicadores de risco calculados. Neste sentido, uma aposta mais determinada e contínua na investigação científica, visando esta temática, constitui um importante desafio societal, pois a obtenção de mais conhecimento nesta área é vital para a obtenção de uma água de abastecimento segura, o que é essencial na proteção e promoção da saúde pública.

No nível da operação, as entidades gestoras dos sistemas de tratamento de água deverão priorizar as suas medidas para a mitigação deste risco, devendo, de acordo com tais resultados, focar-se no aumento de eficácia na redução das concentrações de diclorobromometano e dibromoclorometano que se revelaram parâmetros com influência no valor final de $\mathrm{CR}_{\mathrm{T}}$.

\section{CONCLUSÃO}

Em termos gerais, a qualidade da água distribuída no distrito de Braga cumpre a legislação no que concerne aos limites legais da concentração de trihalometanos na água de abastecimento.

A proporção média da ocorrência das quatro principais espécies de THM na água de abastecimento foi determinada para o distrito de Braga. Os mais abundantes foram o bromofórmio e o clorofórmio com cerca de $30 \%$ cada, parecendo contrariar a noção generalizada de que o clorofórmio é "sempre" a espécie mais representativa. 
A exposição aos THM pela via de ingestão foi confirmada como a mais preponderante no agravamento do risco de cancro total, seguida da inalação e da absorção dérmica, como seria expectável na água para consumo humano.

No quinquénio analisado, os municípios de Amares e de Vila Verde apresentavam os resultados mais preocupantes seguidos pelos de Cabeceiras de Basto e da Póvoa de Lanhoso, sendo o risco de cancro ao longo da vida máximo em Amares e, mínimo, em Terras de Bouro.

Para um cenário extremo, de exposição prolongada a valores da concentração de THM iguais ao valor máximo observado no quinquénio, todos os municípios apresentariam risco superior ao valor de referência definido como negligenciável na metodologia preconizada pela USEPA.

O risco devido aos efeitos nãocarcinogénicos na saúde foi expressivamente mais elevado pela via de exposição por ingestão, em detrimento da via por absorção dérmica, como expectável. Com exceção do município de Amares, o risco de efeitos não-carcinogénicos foi inferior à unidade, indicando, assim, um nível de risco considerado aceitável.

Recomenda-se o desenvolvimento de novos estudos, preferencialmente multidisciplinares, visando o aprofundamento não só da caracterização das variáveis identificadas, como sendo as mais críticas, na análise de sensibilidade efetuada, mas também de metodologias inovadoras de avaliação do risco de cancro, tendo em consideração que, na sua maioria (nomeadamente os associados à exposição a THM), são multicausais.

\section{REFERÊNCIAS}

AMJAD, H.; HASHMI, I.; REHMAN, M. S.; ALI AWAN, M.; GHAFFAR, S.; KHAN, Z. Cancer and non-cancer risk assessment of trihalomethanes in urban drinking water suppliesof Pakistan. Ecotoxicology Environment Safety, v. 91, p. 25-31, mai. 2013.

BASU, M.; GUPTA, S. K.; SINGH, G.; MUKHOPADHYAY, U. Multi-route risk assessment from trihalomethanes in drinking water supplies. Environmental Monitoring and Assessment, v. 178, n. 1-4, p. 121-134, jul. 2011.

DUARTE, A. A. L. S. Impacto das alterações climáticas na gestão da água. Águas \& Resíduos, v. 3, n. 2, p. 58-73, jun. 2007.
ERSAR. Relatório anual dos serviços de águas e resíduos em Portugal (RASARP). Entidade Reguladora dos Serviços de Águas e Resíduos, Lisboa, Portugal, 2012.

Entidade Reguladora dos Serviços de Águas e Resíduos, Lisboa, Portugal, 2013.

Entidade Reguladora dos Serviços de Águas e Resíduos, v. 2, Lisboa, Portugal, 2015.

FLORENTIN, A.; HAUTEMANIERE, A.; HARTEMANN, $P$. Health effects of disinfectionby-products in chlorinated swimming pools. Int J Hyg Environ Health, v. 214 , n. 6 , p. 461-469, nov. 2011.

FU, H. Z.; WANG, M. H.; HO, Y. S. Mapping of drinking water research: Abibliometric analysis of research output during 1992-2011. Science of the Total Environment, v. 443, p. 757-765, jan. 2013.

FUNARI, E.; MANGANELLI, M.; SINISI, L. Impact of climate change on waterborne diseases. Annali dell Istituto Superiore di Sanita, v. 48, n. 4, p. 473-487, abr. 2012.

GOPAL, K.; TRIPATHY, S. S.; BERSILLON, J. L.; DUBEY, S. P. Chlorination byproducts, their toxicodynamics and removal from drinking water. Journal of Hazardous Materials, v. 140, n. 1-2, p. 1-6, fev. 2007.

HANSEN, K. M. S.; WILLACH, S.; ANTONIOU, M. G.; MOSBAEK, H.; ALBRECHTSEN, H. J.; ANDERSEN, H. R. Effect of $\mathrm{pH}$ on the formation of disinfection byproducts inswimming pool water - Is less THM better? Water Research, v. 46, n. 19, p. 6399-6409, set. 2012.

IRIS. Integrated Risk Information System. 2014. Disponível em: http://www.epa.gov/iris. Acesso em: 11 jan. 2019.

KRASNER, S. W. The formation and control of emerging disinfection by-products of health concern. Philosophical Transactions of the Royal Society Mathematical, Physical and Engineering Sciences, v. 367, n. 1904, p. 4077-4095, out. 2009.

LEGAY, C.; RODRIGUEZ, M. J.; SERODES, J. B.; LEVALLOIS, P. Estimation of chlorinationby-products presence in drinking water in epidemiological studies on adverse reproductive outcomes: A review. Science of the Total Environment, v. 408, n. 3, p. 456-472, set. 2010.

LITTLE, J. C. Applying the two-resistance theory to contaminant volatilization in showers. Environmental Science \& Technology, v. 26, n. 7, p. 1341-1349, jul. 
1992.

NIKOLAOU, A.; GOLFINOPOULOS, S.; LEKKAS, T.; ARHONDITSIS, G. Factors affecting the formation of organic by-products during water chlorination: A benchscale study. Water, Air, and Soil Pollution, v. 159, n. 1, p. 357-371, jan. 2004.

PAOPUREE, P.; PANYAKAPO, M.; SOONTORNCHAI, S. Multi-pathway cancer risk assessment of trihalomethanes exposure from chlorinated tap water and indoor swimming pool. In: INTERNATIONAL CONFERENCE ON CHEMISTRY AND CHEMICAL ENGINEERING, 2010, Kyoto, Japão. Proceedings... Kyoto: IEEE, 2010, p. 409-413.

PENTAMWA, P.; SUKTON, B.; WONGKLOM, T.; PENTAMWA, S. Cancer risk assessment from trihalomethanes in community water supply at Northeastern Thailand. International Journal of Environmental Science and Development, v. 4, n. 5, p. 538-544, out. 2013.

RAIS. Risk Assessment Information System. Oak Ridge National Laboratory (ORNL), Institute for Environmental Modeling (TIEM). Knoxville, USA: University of Tennessee (UTK), 2014. Disponível em: http://rais.ornl.gov. Acesso em: 11 jan. 2019.

RICHARDSON, S. D.; PLEWA, M. J.; WAGNER, E. D.; SCHOENY, R.; DEMARINI, D. M. Occurrence, genotoxicity, and carcinogenicity of regulated and emerging disinfection byproducts in drinking water: $\mathrm{A}$ review for research. Mutation Research, v. 636, n. 1-3, p. 178-242, nov. 2007.

RODRIGUES, F. M. Análise do risco da presença de trihalometanos em água para consumo humano, no distrito de Braga. 2014, 116p. Dissertação (Mestrado em Engenharia Civil) - Escola de Engenharia, Universidade do Minho, Braga, Portugal, 2014.

SCHOENY, R. Disinfection by-products: A question of balance. Environmental Health Perspectives, v. 118, n. 11, A466-A467, nov. 2010.

SHARMA, V. K., ZBORIL, R., MCDONALD, T. J. Formation and toxicity of brominated disinfection byproducts during chlorination and chloramination of water: A review. Journal of Environmental Science and Health Part B-Pesticides Food Contaminants and Agricultural Wastes, v, 49, n. 3, p. 212-228, 2014.

USEPA. Exposure Factors Handbook: 2011 Edition. Washington, DC: U.S. Environmental Protection Agency, EPA/600/R-09/052F, 2011, 1436 p.

VIEIRA, J. M. P.; DUARTE, A. A. L. S. Relevância sanitária dos subprodutos da desinfeção da água para consumo humano. Águas \& Resíduos, v. 3, n. 4, p. 38-46, dez. 2007.

VISITAR PORTUGAL. Disponível em: https://www.visitarportugal.pt. Acesso em: 11 jan. 2019. 